\title{
Le théâtre de Thomas Ostermeier : phénomène culturel ou démarche artistique ? Suivi d'un entretien avec Thomas Ostermeier
}

Thomas Ostermeiers Theater: kulturelles Phänomen oder künstlerisches

Konzept? Gefolgt von einem Gespräch mit Thomas OSTERMEIER

Thomas Ostermeier's Theatre: Cultural Phenomenon or Artistic Approach?

Followed by an Interview with Thomas Ostermeier

Jitka Pelechová

\section{OpenEdition}

\section{Journals}

Édition électronique

URL : https://journals.openedition.org/ceg/9110

DOI : $10.4000 /$ ceg. 9110

ISSN : 2605-8359

Éditeur

Presses Universitaires de Provence

Édition imprimée

Date de publication : 1 mai 2013

Pagination : 335-350

ISBN : 0751-4239

ISSN : 0751-4239

\section{Référence électronique}

Jitka Pelechová, « Le théâtre de Thomas Ostermeier : phénomène culturel ou démarche artistique? Suivi d'un entretien avec Thomas Ostermeier », Cahiers d'Études Germaniques [En ligne], 64 | 2013, mis en ligne le 02 janvier 2020, consulté le 02 juin 2021. URL : http://journals.openedition.org/ceg/9110 ; DOI : https://doi.org/10.4000/ceg.9110 


\section{Le théâtre de Thomas Ostermeier : phénomène culturel ou démarche artistique? \\ Jitka PELECHOVÁ \\ Université Paris 10 - Nanterre}

Le metteur en scène Thomas Ostermeier est aujourd'hui l'une des personnalités-phares de la scène culturelle internationale. Ses spectacles sont à l'affiche des grandes institutions, piliers de «l'establishment théâtral » européen, qu'il s'agisse des festivals internationaux (comme celui d'Avignon, dont il fut l'artiste associé en 2004, celui d'Athènes - Épidaure ou celui de Vienne) ou des principales scènes des différents pays (en Allemagne, il dirige la prestigieuse Schaubühne de Berlin et, en France, il est au programme du Théâtre de l'Odéon, du TNT de Toulouse ou du TNB de Rennes, pour ne citer qu'eux). Ses propos sont régulièrement relayés par les médias à large public, des quotidiens nationaux aux chaînes audiovisuelles à grande audience. Son œuvre est récompensée par les distinctions les plus hautes, comme le Lion d'Or à la Biennale de Venise en 2011 ou le titre français d'Officier des Arts et des Lettres en 2010. Bref, tout semble prédestiner Thomas Ostermeier à jouer le rôle d'un représentant majeur de ce que l'on pourrait appeler la culture dominante.

De nombreux professionnels du monde culturel et intellectuel, en Allemagne et au-delà de ses frontières, réservent au théâtre d'Ostermeier un accueil assez froid : il serait, selon eux, l'expression et le résultat d'une politique de communication culturelle, plutôt que le porteur d'une démarche artistique : "La culture c'est la règle, l'art c'est l'exception », selon la formule de Jean-Luc Godard. Mais si vraiment la démarche d'Ostermeier véhicule à tel point les valeurs dominantes, si elle est à ce point en conformité avec l'idéologie culturelle contemporaine, si vraiment elle est si peu pertinente au plan artistique, comment comprendre, au-delà de l'adhésion qu'elle remporte auprès du public, la curiosité qu'elle provoque, et surtout la vigueur des débats qu'elle soulève?

C'est qu'il ne faut pas oublier que le parcours d'Ostermeier est fait de prises de position contestataires face aux pratiques et discours dominants, marqué par la volonté affirmée de se situer en marge, à contre-courant des 
formes et des esthétiques majoritaires. À y regarder de près, donc, l'image que l'on peut avoir de lui comme "phénomène culturel», comme metteur en scène représentant du mainstream esthétique et institutionnel européen contemporain, paraît trompeuse, ou du moins mérite d'être amplement nuancée. Car si l'œuvre du metteur en scène se construit nécessairement, inévitablement, dans la confrontation avec cette culture dominante, il n'en cherche pas moins à construire une vision artistique personnelle, cohérente et indépendante, volontiers en rupture avec les tendances de son temps.

Thomas Ostermeier revient sur certains de ces sujets dans le dialogue que nous retranscrivons ici et que nous avons mené avec lui lors d'une rencontre publique au Théâtre National de Toulouse, le 4 février 2012, à l'occasion des représentations de sa mise en scène d'Othello et dans le cadre du colloque "Contre-cultures à Berlin de 1960 à nos jours - Pratiques alternatives dans les arts ». Dans une partie introductive, nous proposons un résumé condensé des moments et aspects les plus déterminants de cette démarche artistique propre, en nous focalisant sur le parcours professionnel du metteur en scène, et en laissant de côté les éléments de sa biographie personnelle ${ }^{1}$.

Tout d'abord, il y eut au début des années 1990 le choix de la formation à l'École Ernst Busch. Thomas Ostermeier est né et a grandi dans l'ancienne RFA mais, en 1992, il décide d'intégrer cette école prestigieuse, haut lieu de formation et temple de la tradition théâtrale de l'ancienne RDA. L'un des objectifs majeurs de cette école est de former, dans la tradition brechtienne, des artistes socialement responsables et conscients de leur rôle dans la société ; l'apprentissage vise « l'acquisition d'importantes capacités artisticoartisanales et organisatrices, pour former des personnalités artistiques performantes, qui conçoivent leur travail et leur influence publique dans le sens d'une responsabilité socioculturelle $»^{2}$. Le choix d'Ostermeier répond à l'état d'esprit d'un certain nombre de jeunes gens de théâtre ouestallemands, qui à l'époque redécouvrent avec intérêt le théâtre de l'autre côté du Mur : il leur apparait par de nombreux aspects comme une alternative à un certain épuisement esthétique et idéologique de celui qu'ils pratiquent. Ostermeier revendique sans réserve cette logique de rupture avec la tradition thêâtrale ouest-allemande : " Je suis précisément allé à Berlin-Est, à l'École Ernst Busch, pour ne rien devoir à ce théâtre ouest-allemand incarné par Peter Stein $»^{3}$. Notons qu'en citant le nom de Peter Stein, il signale que l'un des grands contestataires du théâtre établi des années 1970 passe pour être

1 Des éléments qui participent naturellement dans une grande mesure à cette construction et attestent du même état d'esprit. Ostermeier lui-même les évoque volontiers dans les entretiens, comme son passé d'objecteur de conscience en signe de révolte contre un père officier militaire, etc.

2 Cf. le site de la Ernst Busch-Schule, www.hfs-berlin.de (consulté le 28 février 2013).

3 « Thomas Ostermeier, scène de générations », conversation entre Thomas Ostermeier et Jean Jourdheuil, in Mouvement, «Spécial Festival d'Avignon », juillet 2001. 
devenu, aux yeux de la jeune génération, l'une des figures de proue de l'establishment théâtral actuel.

Au sortir de cette formation, Thomas Ostermeier se voit confier la direction de la Baracke, scène annexe du Deutsches Theater, l'un des principaux théâtres de l'ex-RDA. Il dirige cette petite scène pendant trois ans, entre 1996 et 1999, et en fait un lieu incontournable de la vie culturelle berlinoise. Il y monte exclusivement des pièces (ultra-)contemporaines, très souvent en création allemande; il est en particulier très attentif à la dramaturgie anglo-saxonne (Mark Ravenhill, Nicky Silver, etc.). Ses spectacles présentent des univers trash, leur esthétique se fonde sur un jeu d'acteur très corporel, nourri par l'héritage de Meyerhold, sur un rythme soutenu, avec généralement un message politique clairement affirmé. Ainsi, selon certains commentateurs, la Baracke s'inscrit dans un rapport d'opposition au Deutsches Theater ${ }^{4}$ car « la démarche meyerholdienne peut être vue ici de manière programmatique : comme l'abandon du style de jeu psychologico-réaliste développé par Stanislavski, cet antagoniste bourgeois de Meyerhold puis de Brecht, et prédominant sur les scènes allemandes $»^{5}$. Mais c'est surtout grâce à l'affirmation d'un répertoire contemporain clairement politique que la Baracke s'adresse à un public différent de celui qui fréquente traditionnellement la maison mère, et que la démarche d'Ostermeier s'oppose aux choix du Regietheater, cette réinterprétation systématique des œuvres classiques par les metteurs en scène, dont le Deutsches Theater est l'un des bastions. Ostermeier résume son opposition aux pratiques théâtrales dominantes de l'époque dans sa conférence de 1999 , qui clôt l'aventure de la Baracke :

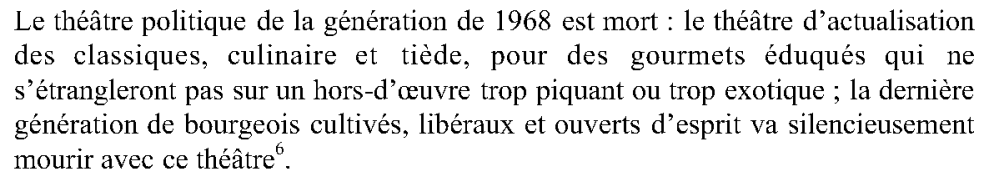

Vient ensuite l'investiture d'Ostermeier à la tête de la Schaubühne am Lehniner Platz, en 1999. Là, à contre-courant des tendances de ce tournant de siècle, en plein essor de l'économie néolibérale qui impose aux théâtres ses lois du marché et du show-business, Ostermeier pose le refus de vedettariat comme fondement du vivre ensemble de la troupe, renouant avec son illustre prédécesseur le jeune Peter Stein. Il gère le collectif selon les principes de la cogestion (Mitbestimmung), répartit la direction entre quatre artistes et fait signer à tous ses acteurs une charte leur interdisant de travailler ailleurs qu'à la Schaubühne (dans d'autres théâtres, au cinéma, à la télévision ou à la radio). Avoir pu et su imposer ce modèle de travail à son collectif relève, à

4 Manuel BRUG, «Die Achtundsechziger kommen », in Der Tagesspiegel, 30 juillet 1997.

5 Cornelia NIEDERMEIER, « Männerspiel. Muskelspiel. », in Die Zeit, 19 février 1998.

6 Thomas OSTERMEIER, «Das Theater im Zeitalter seiner Beschleunigung », in Theater der Zeit, juillet / août 1999 
notre époque, d'un geste contestataire fort ; l'avoir maintenu pendant cinq ans, d'un véritable exploit.

Mais le véritable potentiel contestataire de l'œuvre de Thomas Ostermeier, celui qui provoque les passes d'armes les plus passionnées, réside dans son esthétique et, notamment, dans sa défense du réalisme, forme théâtrale plutôt battue en brèche par les pratiques dominantes de notre temps. Chez le metteur en scène, ce réalisme passe surtout par la mise en avant du récit : il place la narration au centre de sa démarche, et chaque élément de ses représentations semble être mis au service de celle-ci. À une époque dominée par des formes théâtrales qui excèdent volontiers le texte, où les recherches des metteurs en scène (à Berlin comme ailleurs) s'engagent bien souvent dans la voie du théâtre dit «post-dramatique », s'intéresser principalement au récit représente un vrai défi. Pour Ostermeier toutefois, les fables restent essentielles, et doivent détenir une certaine véracité, afin que le public puisse se reconnaître en elles. C'est pourquoi il fait du réalisme son credo esthétique.

Or le seul fait de se réclamer du réalisme aujourd'hui en Allemagne, pays ayant connu les deux grandes dictatures totalitaires du vingtième siècle qui ont, chacune, imposé le réalisme comme l'unique forme artistique valable, passe pour de la provocation. Toutefois, la démarche d'Ostermeier, bien sûr loin de cautionner les idéologies de l'art officiel du Troisième Reich ou du réalisme socialiste, vise au contraire à penser cette démarche artistique en dehors de ces références qui la firent «tomber en discrédit»? S'il veut réhabiliter la notion de réalisme, c'est parce qu'elle est aujourd'hui, selon lui, « employée dans l'objectif de diffamer tel ou tel travail théâtral, ou de faire le tri entre ce qui est de l'avant-garde et ce qui est dépassé ${ }^{8}$. C'est ainsi qu'il souhaite en proposer une forme "nouvelle » et parle d'un "réalisme sociologique $»$ : il s'inscrit dans une logique somme toute assez brechtienne, qui s'applique à mettre au jour certains processus à l'œuvre dans le monde contemporain, à travers notamment l'étude des comportements sociaux, afin de contester, de critiquer et de dénoncer une certaine «involution» idéologique et sociopolitique de notre société actuelle.

Pour véhiculer cette critique et ce questionnement idéologiques et sociopolitiques de la société contemporaine, le metteur en scène est conduit à élargir son répertoire et a de plus en plus souvent recours à l'actualisation des œuvres anciennes ; ceci sans craindre de " traduire » (quitte à les « maltraiter», selon certains) quelques grandes «fables » du répertoire, de Shakespeare à Ibsen, pour les faire coller à sa propre réalité. Or, réinterpréter les pièces classiques par le moyen de la mise en scène, en refusant toute forme de fidélité historique ou littéraire à l'œuvre, dans l'objectif de rapprocher le texte ancien du spectateur contemporain, veut dire renouer avec

7 Sclon Ies propos d'Ostermeier dans son essai « Erkenntnisse über dic Wirklichkeit des menschlichen Mitcinanders. Plädoyer für ein realistisches Theater», in Kräfte messen. Das Körber Studio Junge Regie, Hamburg, éditions Körber-Stiftung, 2009. Citć d'après www.schaubuchne.de (consulté lc 28 février 2013).

$8 \mathrm{Ibid}$. 
la pratique du Regietheater, pratique qu'Ostermeier lui-même contestait dans les années 1990. Cette contradiction a apporté de l'eau au moulin de bien des pourfendeurs de son art, évoqués plus haut. Il ne faudrait pourtant pas appréhender cette évolution en termes de régression idéologique ou de retournement de veste à visée commerciale, comme certains ont tendance à le faire, mais la situer dans le contexte esthétique de son temps : il s'agit, pour Ostermeier, surtout de la défense d'un théâtre "dramatique » devenu marginal et considéré par d'aucuns comme obsolète, ce qui le met en cohérence avec ses revendications premières. À la culture dominante qui tend à ériger en règle la fin des idéologies, de l'Histoire et des grands récits, le metteur en scène oppose une vision artistique faite de récits quotidiens dans lesquels les idéologies et l'Histoire jouent un rôle essentiel, comme dans nos vies.

Il est naturel que tout au long de son parcours et, surtout, au contact de la réalité du travail dans les grandes institutions européennes, le metteur en scène ait été obligé de revoir et relativiser certaines de ses positions contestataires. Mais il n'a pas pour autant abandonné ses principes et engagements idéologiques ou esthétiques essentiels, qui restent présents dans son œuvre : ils lui ont permis d'éclore puis de s'affirmer, et ils continuent à la nourrir.

\section{Rencontre publique avec Thomas Ostermeier, Théâtre National de Toulouse, dans le cadre du colloque «Contre-cultures à Berlin de 1960 à nos jours - Pratiques alternatives dans les arts ", le 4 février 2012.}

Jitka Pelechová : Tu soumets les textes de Shakespeare, comme d'ailleurs toutes les œuvres classiques que tu montes, à un travail d'actualisation assez prononcé. Celle-ci ne se résume pas à une simple transposition des textes dans un milieu contemporain, même s'il y a, bien sûr, des éléments actuels : lorsque tu as monté le Songe d'une nuit d'été de Shakespeare, la pièce se déroulait dans une boîte de nuit contemporaine ; dans Hamlet, la cour d'Elseneur ressemblait à celle, élyséenne, du couple SarkozyBruni ; dans Mesure pour mesure, on nettoyait la racaille au Kärcher... Pour Othello, tu as déclaré au cours des répétitions : « Ce qui m'intéresse, c'est la biographie d'Othello. C'est un arriviste, c'est un homme qui a accédé au pouvoir en partant de sa condition d'esclave, et la classe dominante l'accepte à cause du pouvoir qu'il exerce. Au moment où on n'a plus besoin de lui, on peut se débarrasser de sa présence sans problème ${ }^{9}$. C'était cela, ta porte d'entrée pour ton accès à Othello?

9 Propos tenus dans 1'entretien « La couleur d'Othello doit rester une surprise », in Elefterotypia, 8 mai 2010. 
Thomas Ostermeier : Oui. Au début, je me suis posé la question suivante : dans quelle situation politique se passe cette pièce ? J'ai essayé de trouver des parallèles entre notre situation actuelle et celle de Venise chez Shakespeare. Et j'ai l'impression qu'il y a pas mal d'analogies. Par exemple, cette société vénitienne, assez impérialiste, qui doit faire face à la menace venant d'une autre puissance, mais veut en même temps continuer à faire du commerce, à réaliser du profit et garder le pouvoir économique sur toute la Méditerranée. Il apparaît clairement dans la pièce que les Vénitiens rechignent à aller faire la guerre eux-mêmes : ils engagent donc des mercenaires pour mener leurs combats à leur place, comme Othello, qui a fait carrière dans l'armée vénitienne, ou comme Iago, qui est espagnol et appartient à la même armée. Ce qui m'a interpellé, c'est cette décadence certaine de la société vénitienne qui n'est plus prête à défendre son royaume elle-même. Et tout ça m'a rappelé des situations actuelles comme la guerre d'Irak ou les autres guerres dans le monde...

Il est frappant de voir que le Doge, qui promet à Brabantio de punir celui qui a abusé de sa fille, change de perspective et de discours du tout au tout, dès qu'il apprend qu'il s'agit d'Othello dont il a besoin pour mener sa guerre. Il oublie le crime pour ne penser qu'aux intérêts du pouvoir. À ce moment-là, il se range du côté du Noir, même si ce dernier a enfreint la loi. Je pense donc que ce qui arrive dans la pièce est très lié aux questions de hiérarchie sociale. La jalousie violente de Iago a beaucoup à voir avec le fait qu'il n'a jamais connu de situation confortable dans la société ; il a toujours dû faire ses preuves, être guerrier, être soldat. C'est une perspective très masculine.

Je crois aussi qu'Othello, qui a connu la condition d'esclave, se trouve dans une situation où il a une très grande confiance en sa chance, en sa bonne étoile, car il lui arrive quelque chose qui ne lui est jamais arrivé auparavant. Il sait lutter, il sait combattre, il connaît l'ennemi, il a toutes ces expériences derrière lui, mais il n'a jamais connu d'autre existence. Il n'a jamais rencontré quelqu'un qui, comme Desdémone, l'aime sans réserve. Il sait se défendre, il sait réagir dans une situation où il est attaqué : tuer l'ennemi pour survivre. Mais ici, une sorte de folie s'introduit dans son comportement, ce qui permet à Iago de développer sa stratégie.

Il y a un autre élément qui a aussi été important pour moi : le masque, le fait qu'Othello soit noir. Ma lecture personnelle des pièces de Shakespeare découle toujours du fait que les personnages sont tôt ou tard forcés de jouer un certain rôle dans la société. Un rôle qui n'a rien à voir avec ce qu'ils sont vraiment, avec la vérité cachée derrière la façade. C'est pour ça qu'Othello doit être noir, car il ne joue pas de rôle. Il est le seul qui aime vraiment, qui aime profondément, humainement, qui a des vertus en lui-même. Tout le monde autour de lui est complètement corrompu. Les autres jouent à manifester une certaine idée de l'humanité, ils cherchent à convaincre les autres de se comporter en êtres civilisés. Mais le seul qui est authentiquement humain, c'est Othello. Et tous les autres, notamment Iago qui est appelé tout 
au long de la pièce « l'honnête Iago », sont le contraire de ce qu'on croit d'eux. C'est donc cette idée du masque qui m'a aussi incité à mettre en scène cette pièce, pour montrer que ça n'a pas forcément à voir avec le racisme, mais plutôt avec un jeu de rôle au sein de la société.

JP : Ce jeu de rôle est par ailleurs raconté dans le prologue de ton spectacle, non seulement lorsqu' on dessine un masque noir sur le visage d'Othello mais aussi lorsque son corps nu devient un écran, au sens propre et au sens symbolique, sur lequel les uns projettent leurs angoisses et les autres leurs fantasmes.

Mais si je me souviens bien, ton Othello n'a pas été noir dès la première.

TO : En effet. Et même là, je ne suis pas à cent pour cent convaincu par la solution qu'on a trouvée. Il se pourrait qu'elle évolue encore. Nous jouons cette version pour la sixième fois seulement ; avant, c'était différent. Mais c'est un problème qui, au fond, pour moi, n'en est pas vraiment un, parce que dans mon univers théâtral, dans mon regard sur les personnages, les raisons pour lesquelles Shakespeare a mis le Noir au centre de cette pièce paraissent assez évidentes. Il est vrai qu'aujourd'hui, cela soulève une tout autre discussion; pas seulement sur le racisme dans notre société, mais aussi sur le fait qu'il n'y a pas beaucoup d'acteurs noirs dans les théâtres allemands. Ce débat est très actuel en Allemagne.

Si on regarde la pièce de près, Othello est le seul personnage positif de la pièce, même s'il devient meurtrier à la fin. Tous les autres sont des ordures. Parfois, je me dis qu'Othello est une variation sur quelques éléments qui se trouvent dans d'Hamlet où certains personnages portent des masques pour cacher leur vraie nature ; Hamlet essaie de les démasquer, surtout Claudius et Gertrude. La tragédie dans Othello, c'est qu'au contraire, Othello croit que Desdémone porte un masque, elle aussi : celui d'une femme qui trompe son mari. Mais la réalité est différente. Ce sont les gens autour d'Othello, notamment Iago, qui lui font croire qu'elle est en train de le tromper, mais c'est faux. C'est une pièce qui montre comment Iago peut faire croire à Othello une chose qui n'est pas vraie.

JP : La problématique du regard est en effet au centre de la pièce : ce qu'on voit n'est pas forcément ce qui est, et ce qui est n'est pas forcément ce qu'on voit. Othello demande tout au long de la pièce des preuves de l'infidélité de Desdémone : il veut les « voir».

TO : Et aussi se construire une fausse réalité. Ça a beaucoup à voir avec le théâtre, mais ici, c'est un théâtre "négatif », avec Iago qui met en scène la réalité d'une manière plutôt violente, cynique et terrible. Une fois encore, on revient à Hamlet, où il y a la Souricière ; le théâtre y fait fonction d'instrument «positif », destiné à trouver la vérité. Dans Othello, j'ai l'impression que Shakespeare montre le contraire : le maitre de cérémonie, le 
metteur en scène, Iago, se sert de certains moyens théâtraux pour construire un mensonge.

JP : Je reviens à la question de l'actualisation. Elle passe en grande partie par la scénographie, due à Jan Pappelbaum, qui est ton scénographe principal depuis la fin des années 1990. Il a une formation d'architecte à l'origine - et cela se voit. Comme dans ses autres travaux, il exploite ici aussi tout un vocabulaire architectural, avec des cloisons coulissantes etc. Mais il ajoute à cela un élément extrêmement théâtral, cette eau noire qui se retire, puis revient, en accompagnant la narration de son mouvement. D'un côté, cela permet de transformer le décor en une véritable machine à jouer, au sens meyerholdien du terme, qui détermine le jeu des acteurs et devient un accessoire de jeu pour eux. D'un autre côté, ce bassin semble porteur de références précises : l'or noir, le désert, on a aussi l'impression d'être dans un lieu de vacances des couches aisées de la société ; c'est l'Arabie Saoudite? Las Vegas? Est-ce qu'il y a une référence précise?

TO : On voulait dresser un parallèle dans cette scénographie, et dans l'atmosphère générale de la représentation, entre l'industrie du divertissement et la guerre, ou les guerres, qu'on est en train de mener dans le monde. C'est la raison pour laquelle Iago, chez moi, est plutôt un «entertainer » et que la guerre n'est pas vraiment montrée sur scène. C'est un monde plein de fềte, de musique, de jeu, de divertissement, comme par exemple Las Vegas; un univers qui occupe une place assez importante, surtout dans les sociétés en guerre. L'idée de la scène où Iago et Othello jouent au golf vient d'une photo assez célèbre qui montre George Bush en train de jouer au golf, au moment même où éclate la guerre en Irak. Cette simultanéité des images, des réalités qui, au premier abord, n'ont rien à voir les unes avec les autres, m'interpelle beaucoup. Les images de Hollywood et celles de la guerre font pourtant partie du même monde. J'ai essayé de réunir tout cela dans le spectacle.

JP : Cette transposition participe aussi d'un certain réalisme que tu revendiques pour ton théâtre. Il ne s'agit pas d'un réalisme dans l'esprit du $\mathrm{XIX}^{\mathrm{e}}$ siècle, de type naturaliste, qui voulait représenter une «tranche de vie », photographier la réalité, mais plutôt d'un réalisme au sens de Brecht, pour qui c'est une mise en question des comportements sociaux, qu'il faut représenter par l' " artificialité » propre à l'art. Or on a l'impression, aujourd'hui, que le discours dominant sur les scènes théâtrales contemporaines taxe le réalisme de passéiste, le déclare obsolète, voire tabou, sinon réactionnaire. Est-ce que, d'une certaine manière, se revendiquer du réalisme pourrait être de nos jours un geste contestataire, subversif, révolutionnaire?

TO : C'est une discussion assez allemande et surtout particulièrement déprimante. Depuis dix ou quinze ans, il y a ce débat, ce faux débat, sur la concurrence entre le théâtre "post-dramatique » et le théâtre, disons, 
« dramatique ». La plupart des hommes de théâtre qui mettent encore en scène des histoires, des personnages, un texte, un conflit, risquent d'être considérés par les autres, comme des réactionnaires qui n'ont pas compris que le théâtre, ce n'est plus raconter une histoire, montrer des personnages, les confronter à des situations... À mon avis, cette façon de faire du théâtre, y compris par certains grands maîtres, a été très importante en un temps où déconstruire était une nécessité. Mais moi je me trouve dans une autre situation. À l'époque où je suis venu au théâtre, je me suis senti entouré par des grands frères, comme Christoph Schlingensief, Frank Castorf et d'autres, qui avaient déjà tout déconstruit. Il ne restait plus rien à casser. Il fallait donc bien reconstruire les choses pour qu'un jour, un jeune puisse arriver et tout casser à nouveau. Quant à moi, je suis malheureusement arrivé à un moment où je ne pouvais plus rien casser - c'est dommage, car je crois que mon tempérament me porte plus volontiers à détruire qu'à construire. Mais ça n'aurait pas eu de sens, parce que je serais devenu un épigone; or je ne voulais imiter personne, pas même les grands maîtres.

Pour moi, deux choses sont capitales dans le théâtre : premièrement, m'inspirer des expériences que $\mathrm{j}$ 'ai faites dans ma vie et qui sont liées au monde dont je viens, et deuxièmement, battre en brèche l'idée que pour faire du théâtre, il faut avoir fait des études à l'université plutôt qu'observé la réalité au-dehors : c'est une idée qui m'a toujours profondément irrité. J'essaie de retrouver une certaine forme de narration, car il y a encore beaucoup de conflits à raconter dans le monde. Le théâtre, c'est l'art du conflit. Ceux qui se revendiquent du théâtre «post-dramatique » sont dans une perspective assez cynique : ils prétendent qu'on ne peut plus rien changer car on est arrivé à la fin de l'Histoire, et ça m'énerve. Ça ne correspond absolument pas à notre situation politique actuelle.

Pour ma part, je fais du théâtre pour mieux comprendre ce qui se passe autour de moi et en moi. C'est mon chemin à moi et je suis assez content quand le public partage cet intérêt qui est le mien, pour les pièces, les personnages, les histoires. Alors oui, le réalisme peut être subversif, mais ce n'est pas la raison pour laquelle mon travail a pris cette direction.

JP : Tu viens de créer Mademoiselle Julie d'August Strindberg, à Moscou, au Théâtre des Nations, une grande institution moscovite qui s'est vu attribuer de nouveaux locaux, construits notamment avec l'argent du Kremlin. Tu avais refusé que ton spectacle soit donné en inauguration de ce nouveau théâtre, financé directement par Poutine qui renoue selon toi avec la «tactique du salami » ${ }^{10}$. Mais comme le spectacle qui devait être présenté avant le tien a été annulé au dernier moment, c'est finalement ce qui a eu lieu.

10 Cette expression désigne la manière dont les gouvernements sovićtiques anćantissaient, méthodiquement, l'une après l'autre (" tranche après tranche »), les différentes eatégories d'opposants. Thomas Ostermeier y fait référence dans un entreticn avec Dorte Lena EILERS à propos de son expérience russe, « Hinter den Masken der Macht», in Theater der Zeit, février 2012 . 
Dans ce genre de situation, quel rôle, quelle attitude peut adopter un artiste pour ne pas se faire phagocyter par le système au pouvoir?

TO : D'abord, il faut dire que je ne suis pas un Russe en Russie. Je suis là-bas en visite. Je ne vais pas en Russie pour dire aux Russes comment agir politiquement. J'ai accepté l'invitation parce que le théâtre russe est pour moi l'un des plus vivants, surtout concernant les acteurs (un professeur russe a été très important pour moi pendant ma formation). J'y suis donc allé avec une certaine admiration, mais aussi une certaine distance. Au bout de deux ou trois jours toutefois, je me suis senti en réelle complicité avec les acteurs. Une complicité rare, une complicité avec eux face au pouvoir et à leur situation, qui était à ce moment-là assez délicate. En fait, Evguéni Mironov, mon acteur principal et le directeur du Théâtre des Nations, s'était rapproché de Poutine pour pouvoir construire ce théâtre à Moscou : un théâtre contemporain, avec des metteurs en scène internationaux, sans troupe permanente. C'est rare en Russie, où les grandes institutions sont pour la plupart dirigées par des hommes de théâtre qui ont entre soixante-dix et quatre-vingt-quinze ans. Il n'y a pas de jeunes directeurs de théâtre. C'était donc pour moi un acte de solidarité avec la jeune génération qui essaie de faire quelque chose de différent. Une grande partie des représentations qu'on voit à Moscou sont des spectacles commerciaux, des comédies musicales, ou au contraire des spectacles très muséaux dans leur esthétique. De mon côté, $j$ 'avais proposé une réécriture de Mademoiselle Julie par un auteur russe, Mikhaïl Durnenkov; on s'était dit que cela pouvait être intéressant de mettre à la place de Jean, le domestique de la pièce de Strindberg, un chauffeur chez un oligarque russe, ancien membre du KGB. Nous avons créé le spectacle avec de grandes stars du théâtre et du cinéma russes, nous avons un public très nombreux chaque soir. Il m'a semblé intéressant de lui montrer le regard d'un jeune auteur russe qui a réécrit la pièce à partir de sa situation actuelle, en se focalisant notamment sur l'important décalage entre les riches et les pauvres.

La marge de mon action politique en Russie dépend de mes capacités, qui sont celles d'un metteur en scène. Je ne voulais pas remettre en question tout le projet simplement à cause du fait que je me sois retrouvé à inaugurer le théâtre, même si je l'avais refusé dans un premier temps. Les Russes ont assez de problèmes graves actuellement... Faire du théâtre contemporain, que même les jeunes viennent voir, en Russie, c'est assez rare. Or je crois que c'est très important.

Public : Je vous remercie pour le spectacle que j'ai vu hier et qui est magnifique. Visiblement, vous avez coupé une partie de la fin de la pièce, et je voudrais savoir pourquoi.

TO: J'ai eu l'impression que tous les meurtres qui viennent après celui de Desdémone sont beaucoup trop faibles par rapport à ce premier. Je voulais donc me concentrer sur cette tragédie, pour déboucher sur une situation assez 
surréelle, où Othello comprend ce qu'il a fait et doit continuer de vivre avec. Mais, là encore, il s'agit de la dernière mouture de la mise en scène : il y en avait une précédente, avec tous les autres morts à la fin. On essaie de continuer la recherche pendant les représentations, de ne pas figer le spectacle au sortir des répétitions.

Public : Dans la version allemande, on dit beaucoup der Schwarze. Vous avez traduit par le Black, qui est, à mon sens, moins violent que le Noir. Pourquoi?

TO : Das ist genau der Punkt! Si on avait dit en allemand der Neger, on aurait pu dire en français le Noir ou le nègre. Der Schwarze, en allemand, est une expression assez politiquement correcte. On voulait quelque chose qui ne soit pas explicitement raciste.

Public : Vous dites qu'Othello est le seul personnage de la pièce qui aime vraiment... Et Desdémone? Son amour à elle est plus beau que le sien. Elle dit à Othello : «je t'aime, tu peux faire de moi ce que tu veux »; alors que l'amour d'Othello, c'est : « je t'aime, je peux faire de toi ce que je veux ».

TO : Je suis tout à fait d'accord! Je parlais des personnages masculins qui entourent Othello. Il est vrai que l'amour de Desdémone est tout aussi profond que celui d'Othello. Mais le problème d'Othello, c'est qu'il vit dans un monde masculin - et là, c'est le seul qui aime vraiment. Si on le compare avec Cassio, qui a eu plusieurs maîtresses et qui ne veut pas épouser Bianca, parce que c'est une prostituée, ou avec Iago qui a envers sa femme Emilia un comportement horrible, on comprend qu'Othello est le seul homme dans la pièce qui aime vraiment.

Public : Pourquoi avez-vous décidé de mettre en scène les grands classiques comme Shakespeare, adaptés par Marius von Mayenburg, qui réécrit les œuvres et les transpose dans un langage quotidien?

TO : Ce n'est pas seulement une transposition dans un langage quotidien! D'abord, il faut savoir qu'il n'y pas de véritables manuscrits de Shakespeare. Tout ce dont on dispose a été écrit a posteriori par les acteurs qui avaient joué les rôles ; on n'est même pas sûrs de l'ordre des scènes. La plupart de ses pièces se nourrissent de matériaux préexistants que Shakespeare a relus et adaptés. Il n'existe donc pas de texte original. Chaque traduction est une interprétation particulière. Il faut constamment faire des choix de traduction lorsqu'on met en scène Shakespeare. Avec Marius, on essaie d'être très près du sens et du contenu de la pièce. C'est notre objectif principal. Il ne s'agit pas de faire une modernisation, mais plutôt de mieux comprendre ce que la scène raconte, avec une traduction en prose - nous ne respectons pas le 
blankvers (vers blanc) de Shakespeare. C'est plutôt un travail de recherche que d'adaptation. On cherche à comprendre de quoi les pièces parlent.

Public : Parfois, on a l'impression que ça devient plus violent.

TO : Le langage de Shakespeare lui-même (si ce qu'on a est réellement de Shakespeare) est déjà très violent.

Public : J'ai une question concernant la scénographie. J'aimerais savoir quelle signification ont le bassin d'eau et les cloisons verticales qui laissent une certaine transparence. Qu'est-ce que ça apporte à la pièce ?

TO : C'est tout ce que vous voulez. Cela pourrait évoquer Venise, avec l'eau noire, mais aussi le pétrole... La signification n'est pas univoque et arrêtée. Il y a plusieurs possibilités et c'est à vous, les spectateurs, de décider ce que vous voulez y voir. Ce n'est pas une scénographie réaliste, plutôt un terrain de jeu.

Public : Ma question concerne la direction d'acteurs. Est-ce que les acteurs sont choisis en fonction de l'idée que vous vous faites du rôle, ou estce que le rôle est construit à partir des personnalités et des propositions des acteurs?

TO : C'est une question compliquée. D'abord il faut savoir que la Schaubühne dispose d'une troupe fixe. Il y a vingt-huit acteurs permanents. Tous les acteurs dans Othello font partie de la troupe, il n'y pas d'invités pour ce spectacle. Normalement, je cherche des pièces et des rôles pour mes acteurs de la Schaubühne. Il y a Stefan Stern - et un jour, je me dis : Tiens, il pourrait jouer Iago. Et c'est comme ça que je décide de monter la pièce. Il y a donc d'abord l'acteur, et après le rôle.

Public : Pourquoi les acteurs restent-ils sur scène tout au long du spectacle?

TO : Je n'aime pas quand les acteurs, après avoir joué une scène très chargée en émotions par exemple, partent à la cafétéria pendant une heure et reviennent après, en essayant de rentrer à nouveau dans le spectacle... Je pense que tous les acteurs sont responsables de la représentation, même lorsqu'ils ne parlent pas. Mais c'est également très lié à l'écriture de Shakespeare : si les acteurs restent en scène, on peut avoir des enchaînements beaucoup plus rapides et accélérer le rythme du spectacle.

Public : J'ai une question un peu extérieure à la pièce. Je me demandais comment était perçue l'importance du théâtre et sa valeur avant et après la réunification de Berlin? 
TO : Il faudrait faire un discours de deux ou trois heures à ce sujet... Une question gigantesque. Aïe aïe aïe...

JP : Je me propose de t'aider, si tu permets... Maurice Taszman, qui est dans le public, a prononcé une phrase qui, je pense, répond bien à cette question. Parlant des raisons pour lesquelles Frank Castorf, lorsqu'il a pris la direction de la Volksbühne à Berlin, a fait inscrire sur le toit du théâtre, en énormes lettres de néon, le mot Ost (Est), il a suggéré qu'il s'agissait d'affirmer que le théâtre, c'est ce qui est resté de l'Allemagne de l'Est après la chute du Mur. Le théâtre a été l'unique terrain à échapper à la colonisation de l'Est par l'Ouest.

Public : Est-ce que vous avez été, ou êtes-vous encore aujourd'hui, acteur? Ou bien vous consacrez-vous uniquement à la mise en scène ?

TO : Oui, j'ai été acteur au Berliner Ensemble, à l'époque où Heiner Müller était encore vivant. Je me souviens que nous avions fait une « soirée Brecht » avec Manfred Karge, à partir des chansons et des textes politiques de Brecht. Heiner Müller était venu voir la générale, et après, il avait demandé à Manfred Karge : « Ce jeune-là, il vient d'où ? De l'Est ou de l'Ouest?» Karge lui avait répondu que je venais de l'Ouest. Et Müller avait dit alors : «Dans ce cas, il ne peut pas jouer Brecht». Je me suis senti un peu offusqué, car je suis originaire de Bavière et je pense qu'il faut avant tout venir de Bavière pour pouvoir jouer Brecht...

Jitka Pelechová est l'auteur d'une importante thèse intitulée Le théâtre de Thomas Ostermeier: en quête d'un réalisme nouveau qui paraîtra à l'automne 2013 aux éditions Études théâtrales. Elle est dramaturge de plateau et traductrice. 